\title{
MODIFIED SPECTRAL METHOD OF ANISOTROPIC TURBULENT VELOCITY FIELD GENERATION PRESERVING INCOMPRESSIBILITY
}

\author{
ANATOLY V. ALEXANDROV ${ }^{1}$, LUDWIG W. DORODNICYN ${ }^{2}$, ALEXEY P. DUBEN ${ }^{3}$ \\ AND DMITRIY R. KOLYUKHIN ${ }^{4}$ \\ ${ }^{1}$ Keldysh Institute of Applied Mathematics RAS, \\ Miusskaya sq., 4, Moscow, 125047, Russia \\ anatoly.v.alexandrov@gmail.com, http://caa.imamod.ru/ \\ ${ }^{2}$ M.V. Lomonosov Moscow State University, \\ Lenin Hills, 1, Moscow, 119991, Russia \\ dorodn@cs.msu.su, https://www.msu.ru \\ ${ }^{3}$ Keldysh Institute of Applied Mathematics RAS, \\ Miusskaya sq., 4, Moscow, 125047, Russia \\ dualks@gmail.com, http://caa.imamod.ru,http://caa.imamod.ru/ \\ ${ }^{4}$ Trofimuk Institute of Petroleum Geology and Geophysics SB RAS, \\ Novosibirsk, Russia, Koptugave. 3, Novosibirsk, Russia, 630090, \\ KolyukhinDR@ipgg.sbras.ru, http://www.ipgg.sbras.ru.
}

Key words: Synthetic turbulent fields, RANS/LES.

\begin{abstract}
A method for the numerical generation of anisotropic turbulent velocity fields is presented. The proposed technique is based on the spectral method (SM) [1]. The traditional adaptation of isotropic field generated with spectral methods uses a Cholesky decomposition of Reynolds stresses tensor. After this adaptation the resulted field loses the property of incompressibility provided in the isotropic case. We have modified this method to use it in the anisotropic case and guarantee the incompressibility of generated turbulent field. Comparison of the results of IDDES simulation of canonical turbulent flow using inlet boundary conditions based on modified and non modified spectral methods are presented.
\end{abstract}

\section{INTRODUCTION}

Synthetic turbulent fields are widely used to generate artificial inflow for ReynoldsAveraged Navier-Stokes (RANS)/Large-Eddy-Simulation (LES) applications.

It's natural to believe that the quality and usefulness of the generators of synthetic turbulence depends on how realistically we can recreate a given physical flow field. Usage of unrealistic turbulent fields at the inflow to the LES zone can require a long spatial distance to develop into realistic turbulence or can lead to significant and even total dissipation of turbulent pulsations [2]. Although there is no exhaustive understanding of what requirements 
for obtained fields can guarantee their realism but, usually, they believe that among key properties of the artificial turbulent fields there should be incompressibility.

There are several approaches for the numerical generation of turbulent velocity fields are exist. According [3,4] among them are the Fourier modes method, synthetic eddy methods and digital filter methods. The first attempts to synthesize an artificial turbulent field were made fifty years ago. Kraichnan [5] has presented a simple in realization version of the Fourier modes method. Following this approach a turbulent field can be synthesized through the summation of Fourier modes. Within the next years, the proposed method was significantly improved [1,6-8]. According to the modern version of the Kraichnan-type method $[1,8]$ the turbulent velocity field is usually built in two stages. At the first stage, a homogeneous isotropic incompressible turbulent field is synthesized as a sum of Fourier cosine modes on the base of the given von Karman spectrum of turbulence. At the second stage, in order to obtain anisotropic turbulent field the obtained isotropic field should be scaled with Cholesky decomposition of Reynolds stresses or with the technique proposed in [9]. After this scaling the resulted field loses the property of incompressibility.

In this paper we present a method of adaptation of isotropic fields obtained at the first stage of spectral methods, preserving incompressibility in anisotropic case.

Comparison of the results of IDDES simulation of canonical turbulent flow using inlet boundary conditions based on modified and non modified spectral methods are presented.
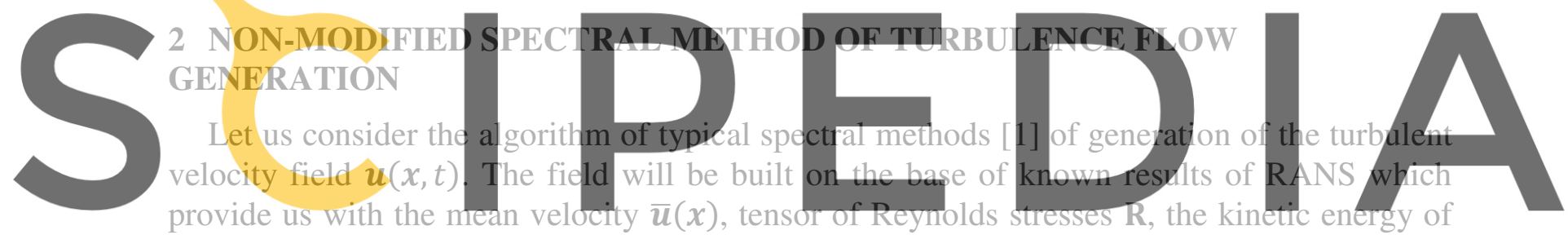
turbulence $\sigma^{2}$ and rate of its dissipation $\varepsilon$. As usual, the instantaneous velocity $u(x, t)$ will be

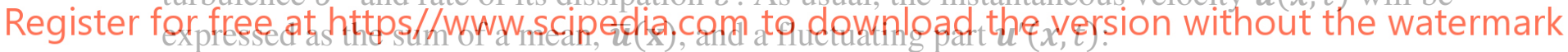

$$
u(x, t)=\bar{u}(x)+u^{\prime}(x, t) .
$$

For simplicity, we will not consider the time dependence below. The method described here can be easily extended using the time dependence described in [1].

An isotropic incompressible turbulent field is synthesized as a sum of Fourier cosine modes:

$$
\boldsymbol{v}^{\prime}(\boldsymbol{x})=2 \sqrt{\frac{3}{2}} \sum_{n=1}^{N} \sqrt{q^{n}} \boldsymbol{\sigma}^{n} \cos \left(k^{n}\left(\boldsymbol{\omega}^{n} \cdot \boldsymbol{x}+\varphi^{n}\right) .\right.
$$

Here $N$ is the number of modes,

$q^{n}$ is the normalized squared amplitude of the mode $n$ defined by the local energy spectrum,

$k^{n}$ is the wave vector absolute value (wave number),

$\boldsymbol{\omega}^{n}$ is a direction vector for the wave vector $\left(\boldsymbol{k}^{n}=k^{n} \boldsymbol{\omega}^{n}\right)$ which is uniformly distributed over the unit sphere,

$\boldsymbol{\sigma}^{n}$ is a unit vector orthogonal to $\boldsymbol{\omega}^{n}$ uniformly distributed by the angle,

$\varphi^{n}$ is a phase of the mode which is a random number uniformly distributed in the interval $[0,2 \pi)$. 
Wave numbers $k^{n}$ are equidistantly distributed by an exponential law over the range:

$$
k^{n}=k_{\min }(1+\alpha)^{n-1}, \quad n=1,2, \ldots, N \text {. }
$$

The normalized amplitudes of the modes $q^{n}$ following [1] are defined as:

$$
q^{n}=\frac{E\left(k^{n}\right) \Delta k^{n}}{\sum_{n=1}^{N} E\left(k^{n}\right) \Delta k^{n}}, \quad \sum_{n=1}^{N} q^{n}=1
$$

where $E\left(k^{n}\right)$ is a modified Karman-Pao energy spectrum:

$$
E(k)=\frac{\left(k / k_{e}\right)^{4}}{\left(1+2.4\left(k / k_{e}\right)^{2}\right)^{17 / 6}} f_{\eta}(k) f_{c u t}(k) .
$$

Here $k_{e}$ is the wave number corresponding to the spectral energy maximum.

The component $f_{\eta}(k)$ provides asymptotics at Kolmogorov scale:

$$
f_{\eta}(k)=\exp \left[-\left(12 k / k_{\eta}\right)^{2}\right] .
$$

The component $f_{\text {cut }}(k)$ ensures dumping at the scale of the mesh cell:

$$
f_{\text {cut }}(k)=\exp \left(-\left[\frac{4 \max \left(k-0.9 k_{c u t}, 0\right)}{k_{c u t}}\right]^{3}\right) \text {. }
$$

The incompressibility of such isotropic field is provided by the orthogonality of unit vector $\boldsymbol{\sigma}^{n}$ to the wave vector $\boldsymbol{\omega}^{n}$.

At the second stage of the spectral methods the Cholesky factorization of the Reynolds

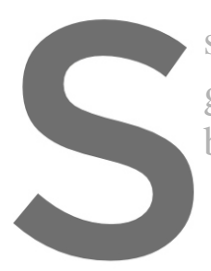
stress tensor $\mathbf{R}=\overline{\mathbf{A}} \overline{\boldsymbol{A}}^{T}$ generated field to the $\mathbf{R}$ be built as

Unfortunately, a
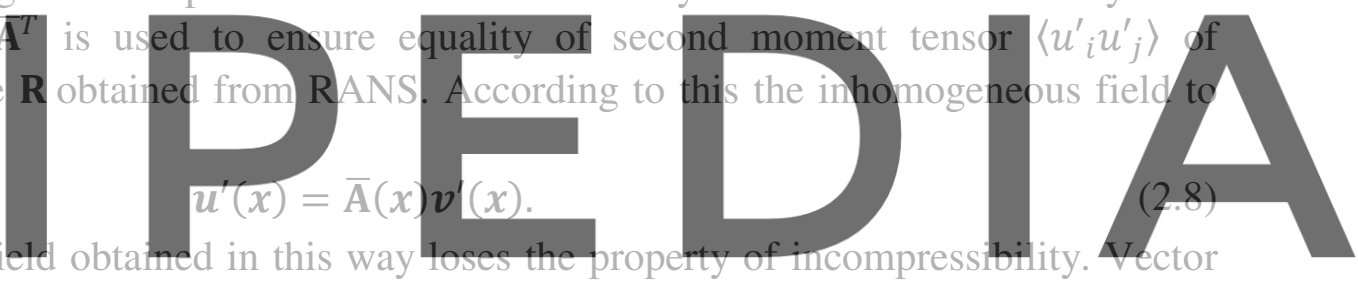

field $v^{\prime}(x)$ satisfies the equation div $v^{\prime}=0$. However, linear transformation (2.8) affects the

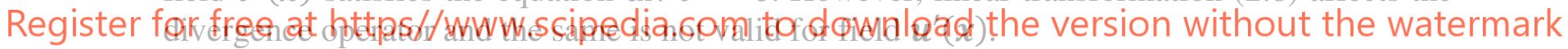

\section{DIVERGENCE-FREE ANISOTROPIC MODIFICATION}

In order to preserve the incompressibility of a velocity field, we will revise the principle of its construction. Based on the ideas by $[10,11]$, instead of dealing with the Reynolds stress tensor, we turn to the spectral description of turbulence. Thereafter we proceed to averaged quantities again, however, the result will be different. In particular, not tensor $\mathbf{R}$ but rather an auxiliary matrix $\mathbf{M}_{\mathbf{R}}$ will be obtained and factorized for the velocity field generation.

Spectral homogeneous turbulence theory [12] relies upon the notion of full spectral covariance tensor $\Phi_{i j}(\boldsymbol{k})$ (here $\boldsymbol{k}$ is wave vector) which is Fourier dual to the tensor of spatial covariances of velocity perturbations. Tensor $\boldsymbol{\Phi}(\boldsymbol{k})$ implies all the other spectral and averaged characteristics of a turbulent velocity field.

Among the properties of tensor $\boldsymbol{\Phi}(\boldsymbol{k})$ emphasize its symmetry and non-negativity

$$
\Phi_{i j}=\Phi_{j i}, \quad \boldsymbol{\Phi}(\boldsymbol{k}) \geq 0, \forall \boldsymbol{k} .
$$


Besides, as the turbulent velocity field is incompressible, consequently the orthogonality condition holds:

$$
\Phi_{i j} k_{j}=0 \text {. }
$$

Spectral energy density tensor $\boldsymbol{\Psi}(k)$ is defined as the integral of $\boldsymbol{\Phi}(\boldsymbol{k})$ over the sphere of radius $k$ :

$$
\Psi_{i j}(k)=k^{2} \int \Phi_{i j}(k \boldsymbol{\omega}) d^{2} \omega, \boldsymbol{k}=k \boldsymbol{\omega},|\boldsymbol{\omega}|=1 .
$$

Here $k$ is the wave number and $\boldsymbol{\omega}$ the direction of wave vector.

The trace of $\boldsymbol{\Psi}(k)$ matrix equals double spectral energy density

$$
\Psi_{i i}(k)=2 E(k) \text {. }
$$

The basic averaged characteristics of turbulence-Reynolds stress tensor $R_{i j}$ and turbulent kinetic energy $\sigma^{2}$-are obtained as the following integrals over all wave numbers:

$$
\begin{aligned}
& R_{i j}=\left\langle u_{i} u_{j}\right\rangle=\int_{0}^{\infty} \Psi_{i j}(k) d k, \\
& \sigma^{2}=\frac{1}{2}\left\langle u_{i} u_{i}\right\rangle=\int_{0}^{\infty} E(k) d k .
\end{aligned}
$$

The present study deals with an inverse problem: knowing Reynolds stress tensor $\mathbf{R}$ and energy spectrum $E(k)$ we should obtain an appropriate full spectral tensor $\Phi(k)$. Apart from the classical postulates stated above, in our procedure of constructing urbulent velocity field two simplification hypotheses
First, suppose there exist sy
and full spectral tensor $\Phi(\boldsymbol{k})$
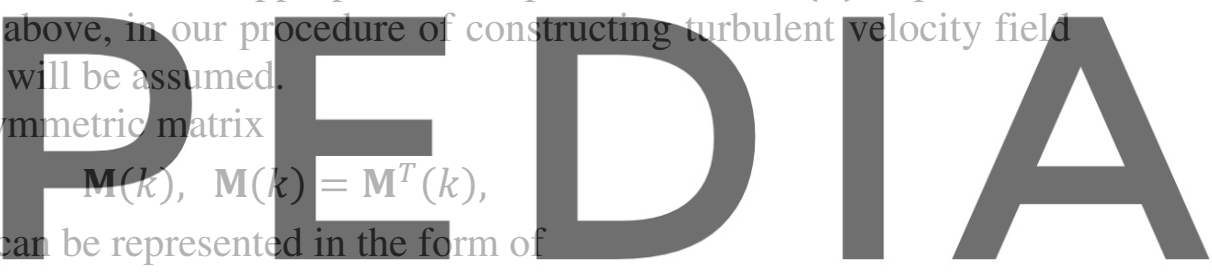

$$
\Phi(k)=\mathbf{Q}(\omega) \mathbf{M}(k) \mathbf{Q}(\omega) .
$$

(3.4)

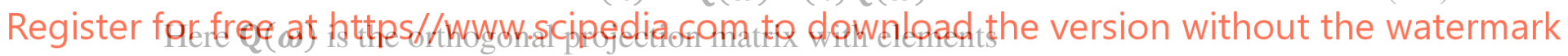

$$
Q_{i j}(\omega)=\delta_{i j}-\omega_{i} \omega_{j}
$$

List the important properties of matrix $\mathbf{Q}=\mathbf{Q}(\boldsymbol{\omega})$ below:

$$
\mathbf{Q}=\mathbf{Q}^{T} \geq 0, \mathbf{Q}^{2}=\mathbf{Q}, \quad Q_{i j} \omega_{j}=0 .
$$

A detailed substantiation to expression (3.4) can be found in [13]. Such tensor $\boldsymbol{\Phi}(\boldsymbol{k})$ does evidently satisfy the orthogonality condition (3.1).

The second assumption concerns a relation of spectral density tensor $\Psi_{i j}(k)$ to Reynolds stress tensor $R_{i j}$. We will use the simplest model which is implicitly accepted by most authors of synthetic turbulence generators [1,9] and states the equal anisotropy over all spectrum

$$
\Psi_{i j}(k)=\frac{E(k)}{\sigma^{2}} R_{i j}
$$


Under assumption (3.4), the procedure (3.2) of obtaining tensor $\boldsymbol{\Psi}(k)$ from $\boldsymbol{\Phi}(\boldsymbol{k})$ can be carried out analytically - see the details in [13,14]. The integration of (3.4) over all directions $\boldsymbol{\omega}$ results in the following expressions for matrix $\boldsymbol{\Psi}(k)$ and its trace:

$$
\begin{gathered}
\Psi_{i j}(k)=\frac{4 \pi k^{2}}{15}\left(7 M_{i j}+\operatorname{tr}(\mathbf{M}) \delta_{i j}\right), \\
\operatorname{tr}(\boldsymbol{\Psi})=\frac{8 \pi k^{2}}{3} \operatorname{tr}(\mathbf{M}) .
\end{gathered}
$$

Using Eq. (3.6) we are able to solve the inverse problem of expressing tensor $\mathbf{M}(k)$ through tensor $\boldsymbol{\Psi}(k)$ :

$$
M_{i j}(k)=\frac{1}{56 \pi k^{2}}\left(30 \Psi_{i j}(k)-3 \operatorname{tr}(\boldsymbol{\Psi}) \delta_{i j}\right)
$$

The task of constructing a full spectral tensor $\boldsymbol{\Phi}(\boldsymbol{k})$ on the base of Reynolds stress tensor $\mathbf{R}$ becomes much simpler owing to assumption (3.5). Let us introduce tensor $\mathbf{M}_{\mathbf{R}}=\left\{M_{i j}^{\mathrm{R}}\right\}$ independent of wave number as the integral of $\mathbf{M}(k)$ over all spectrum:

$$
M_{i j}^{\mathrm{R}}=\int_{0}^{\infty} M_{i j}(k) d k \text {. }
$$

Equation (3.7) implies the expression for this tensor

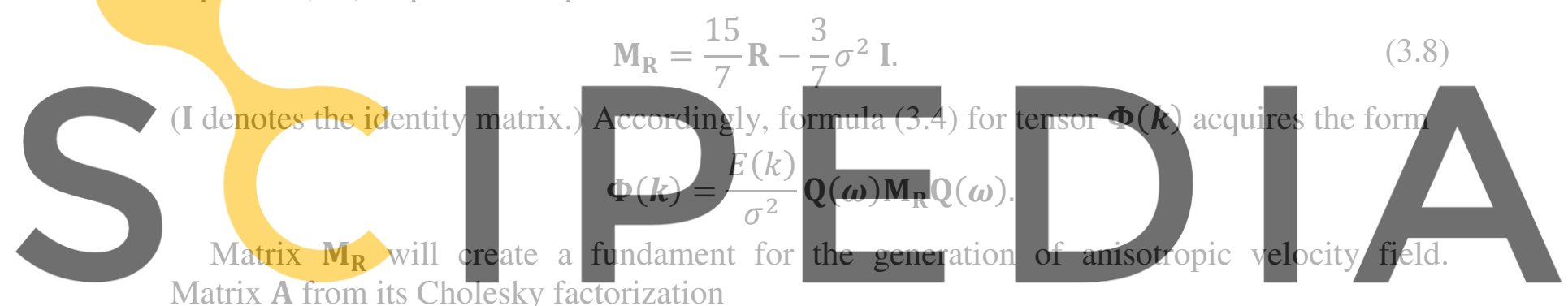
Matrix A from its Cholesky factorization

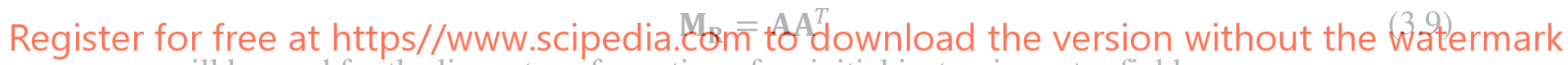
will be used for the linear transformation of an initial isotropic vector field.

We should clarify the question whether tensor $\mathbf{M}_{\mathbf{R}}$ is positive. By taking into account the fact that both tensors $\mathbf{R}$ and $\mathbf{M}_{\mathbf{R}}$ are symmetric, one can obtain (details available in [13]) from Eq. (3.8) the bounds for matrix $\mathbf{R}$ to ensure the positivity of $\mathbf{M}_{\mathbf{R}}$, namely,

$$
\frac{1}{10} \operatorname{tr}(\mathbf{R}) \mathbf{I} \leq \mathbf{R} \leq \frac{4}{5} \operatorname{tr}(\mathbf{R}) \mathbf{I}
$$

The latter requirement is not too restrictive. Experimental measurements of the Reynolds stresses in various turbulent flows (see, e.g., [15]) expose much lower anisotropy than that determined by this inequality.

An anisotropic synthetic turbulent field is generated according to the formula

$$
\boldsymbol{u}^{\prime}(\boldsymbol{x})=2 \sqrt{\frac{3}{2}} \sum_{n=1}^{N} \sqrt{q^{n}} \mathbf{Q}\left(\boldsymbol{\omega}^{n}\right) \mathbf{A} \xi^{n} \cos \left(k^{n}\left(\boldsymbol{\omega}^{n} \cdot \boldsymbol{x}\right)+\varphi^{n}\right) .
$$

The notation is similar to Eq. (2.2), while $\xi^{n}$ is a direction vector uniformly distributed over the unit sphere.

The energy spectral density $E(k)$ is assumed to obey the modified Karman-Pao law (2.5) (2.7) as well as in the isotropic case. 
To make the field divergence-free and obeying the statistics given by the Reynolds stress tensor specified, each mode $\boldsymbol{v}^{n}(\boldsymbol{x})$ is subject to a linear transformation

$$
\boldsymbol{u}^{n}(\boldsymbol{x})=\mathbf{Q}\left(\boldsymbol{\omega}^{n}\right) \mathbf{A} \boldsymbol{v}^{n}(\boldsymbol{x}), \quad \boldsymbol{v}^{n}(\boldsymbol{x})=\boldsymbol{\xi}^{n} \cos \left(k^{n}\left(\boldsymbol{\omega}^{n} \cdot \boldsymbol{x}\right)+\varphi^{n}\right) .
$$

Here the triangular matrix $\mathbf{A}$ is obtained from matrix $\mathbf{M}_{\mathbf{R}}$ factorization (9):

$$
\mathbf{A}=\left\{a_{i j}\right\}=\left(\begin{array}{ccc}
\sqrt{M_{11}^{\mathrm{R}}} & 0 & 0 \\
M_{21}^{\mathrm{R}} / a_{11} & \sqrt{M_{22}^{\mathrm{R}}-a_{21}^{2}} & 0 \\
M_{31}^{\mathrm{R}} / a_{11} & \left(M_{32}^{\mathrm{R}}-a_{21} a_{31}\right) / a_{22} & \sqrt{M_{33}^{\mathrm{R}}-a_{31}^{2}-a_{32}^{2}}
\end{array}\right) .
$$

Although, in the procedure presented, the scaling is applied not to a vector field as a whole but rather to each Fourier mode separately, matrix decomposition (9) is common for all the modes. This factorization of the 3-by-3 matrix is performed once in the case of homogeneous turbulence and at each boundary mesh node for the inhomogeneous case.

Remark that the isotropic version of the velocity field (3.10) will not coincide with Eq. (2.2) due to the distinction in obtaining a direction orthogonal to the wave vector.
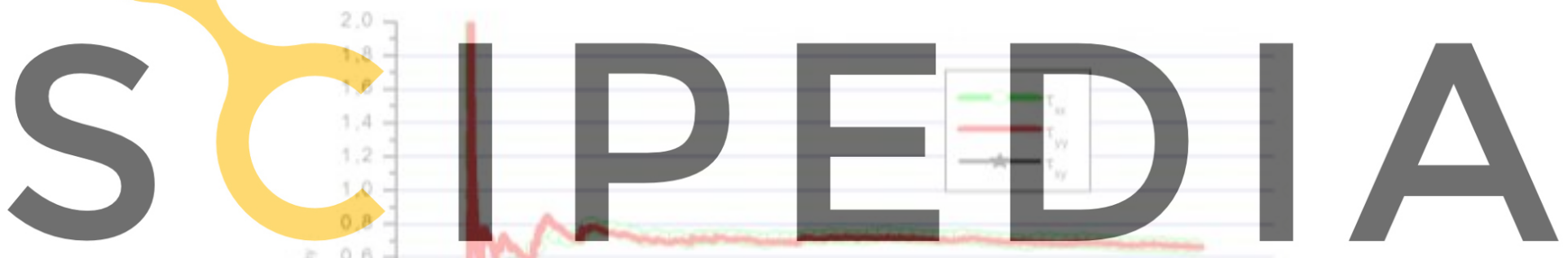

Register for free at https//www.scipedia.com to download the version without the watermark

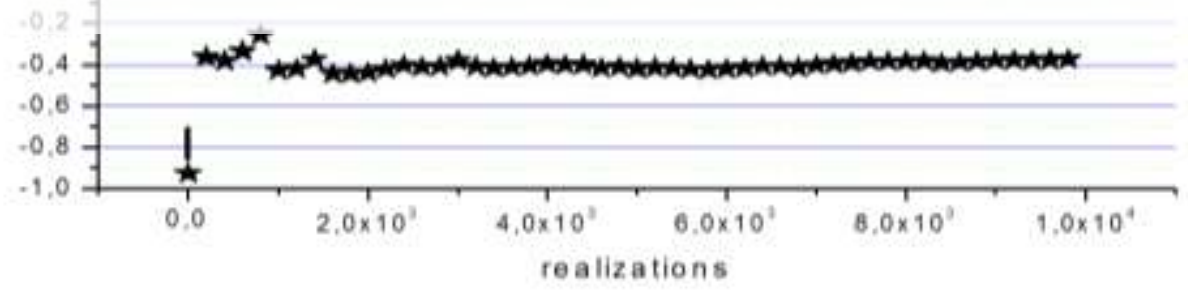

Figure 1: Components of the Reynolds stress tensor of generated fields.

\section{NUMERICAL VERIFICATION AND VALIDATION}

In order to verify numerically the method presented we estimated fields generated on the example of Reynolds stress tensor $\mathbf{R}$ :

$$
\mathbf{R}=\frac{2}{15}\left(\begin{array}{ccc}
5 & -2.8 & 0 \\
-2.8 & 5 & 0 \\
0 & 0 & 5
\end{array}\right) \approx\left(\begin{array}{ccc}
0.667 & -0.373 & 0 \\
-0.373 & 0.667 & 0 \\
0 & 0 & 0.667
\end{array}\right)
$$


The corresponding components of tensor $\mathbf{R}$ obtained on the base of numerically generated velocity fields are presented in Figure 1. Easy to see that obtained values of components of $\mathbf{R}$ are close to the original Reynolds stress tensor.

The numerical validation of the presented modification was performed on the base of a hybrid RANS-LES [8] simulation of turbulent flow over a flat plate with $\operatorname{Re}_{\theta}=7200$. For this problem it is possible to use RANS results as a reference.

The numerical domain was a parallepiped measuring $\{-0,991 \mathrm{c}<x<0\} \times\{0<y<c\} \times$ $\{-0,1 \mathrm{c}<z<0,1 \mathrm{c}\}$. At the left border of domain the inflow boundary conditions based on the generated artificial turbulent fields were prescribed. The Reynolds stress tensor for it was obtained from RANS simulation with Spalart-Allmaras turbulence model [16].

The simulation was performed with and Edge-based reconstruction schemes for unstructured tetrahedral meshes EBR4 [17]. For the simulation the parallel in-house research CFD\&CAA code NOISEtte [18] was used. The calculations were carried out on the hybrid supercomputer K60 located at the Supercomputer Centre of Collective Usage of KIAM RAS.

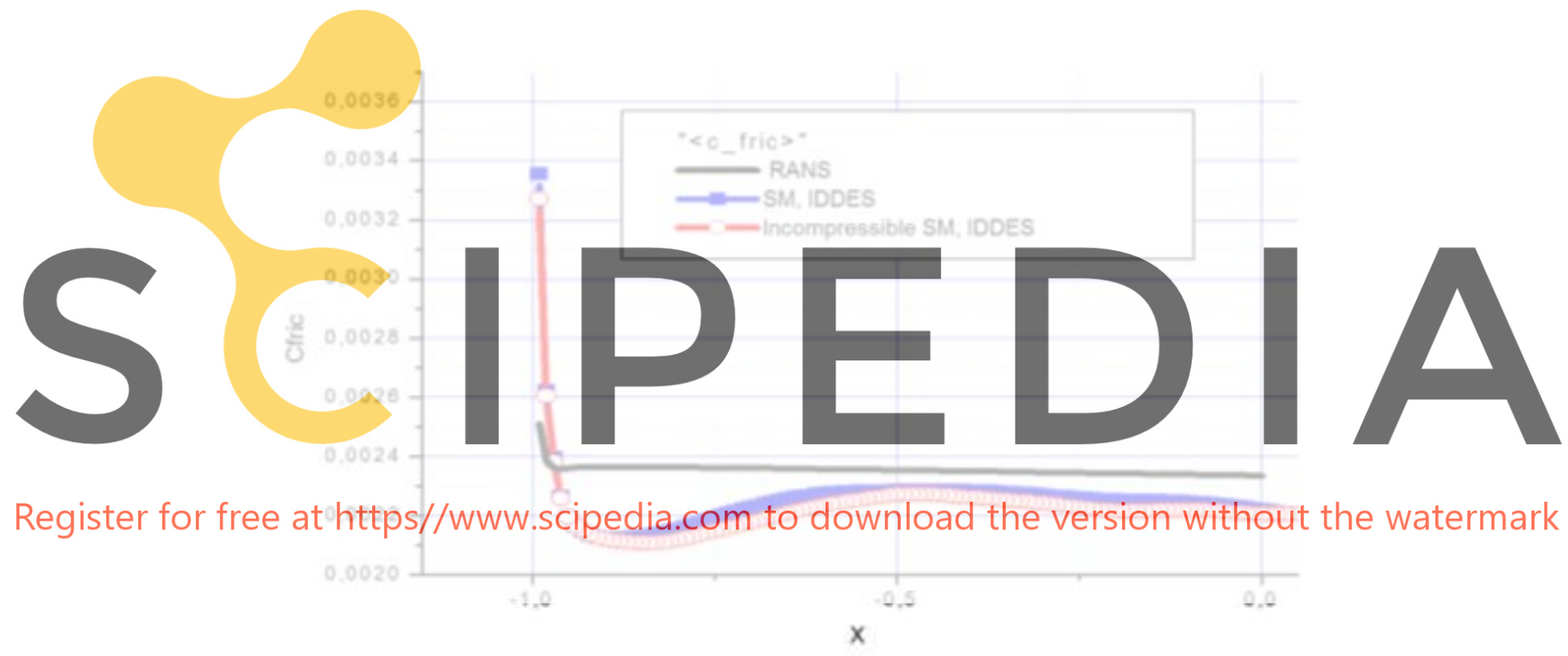

Figure 2: Skin friction coefficients obtained with RANS, non modified spectral method and modified spectral method

In Fig. 2 the skin friction coefficients, obtained with RANS, non modified spectral method (SM) and described incompressible spectral method (ISM) are presented. It can be seen that the results of SM and ISM are close to each other. Adaptation length is the same for both methods. 


\section{CONCLUSION}

The presented method of adaptation of artificial isotropic velocity field for anisotropic case preserves incompressibility. Validating simulation of turbulent flow over a flat plate with boundary conditions based on the proposed algorithm demonstrates that obtained results are not interior the traditional ones obtained with Cholesky decomposition of Reynolds stress tensor. At the same time, it can be expected that this method will have advantages in problems where the incompressibility of a turbulent field is important.

\section{REFERENCES}

[1] Shur, M.L., Spalart, P.R., Strelets, M.K., and Travin, A.K. Synthetic turbulence generators for RANS-LES interfaces in zonal simulations of aerodynamic and aeroacoustic problems. Flow Turbulence Combust. (2014) 93(1):63-92.

[2] Dhamankar, N.S., Blaisdell, G.A., and Lyrintzis, A.S. Overview of turbulent inflow boundary conditions for Large-Eddy Simulations. AIAA J. (2018) 56(4):1317-1334.

[3] Wu, X. Inflow turbulence generation methods. Annual Review of Fluid Mechanics (2017) 49:23-49.

[4] Tabor, G.R. and Baba-Ahmadi, M.H. Inlet conditions for large eddy simulation: A review, Computers and Fluids (2010) 39(4):553-567.
Kraichnan, R. Diffusion by a random velocity field. Phys. Fiuids (1970) 13(1):22-31.
Bechara, W., Bailly, C., Lafon, C., and Candel, S.M. Stochastic approach to noise
modeling for free turbulent flows. AIAA J. (1994) 32(3):455-463.
Saad, T., Cline, D., Stoll, R., and Sutherland, R. Scalable tools for generating synthetic iso-tropic turbulence with arbitrary spectra. AIAA J. (2016) 55(18):327-331.

Register for free at https//www.scipedia.com to download the version without the watermark

[8] Shur, M.L., Spalart, P.R., Strelets, M.Kh., and Travin, A.K. A hybrid RANS-LES approach with delayed-DES and wall-modeled LES capabilities. Int. J. Heat Fluid Flow (2008) 29(6):1638-1649.

[9] Smirnov, A., Shi, S., and Celik, S., Random flow generation technique for Large Eddy Simula-tions and Particle-Dynamics Modeling. J. Fluids Eng. (2001) 123(2):359-371.

[10] Sabelfeld, K.K. Monte Carlo Methods in Boundary Value Problems. Springer, Heidelberg-Berlin-New York, (1991).

[11] Kurbanmuradov, O., Sabelfeld, K., and Kramer, P.R. Randomized spectral and Fourierwavelet methods for multidimensional Gaussian random vector fields. J. Comput. Phys. (2013) 245:218-234.

[12] Batchelor, G.K. The Theory of Homogeneous Turbulence. Cambridge University Press, London-New York, (1959).

[13] Alexandrov, A., Dorodnicyn, L., and Kolyukhin, D. Stochastic algorithm for generation of a divergence-free anisotropic homogeneous turbulent velocity fluctuation field. Preprint Keldysh Inst. Applied Math., Moscow, (2020) 119:17p. 
[14] Alexandrov, A.V., Dorodnicyn, L.W., Duben, A.P., and Kolyukhin, D.R. Generation of a random anisotropic incompressible turbulent velocity field in accordance with the calculated flow statistics. J. Phys.: Conf. Ser. (2021) 1715(012059):1-7.

[15] Stiperski, I. and Calaf, M. Dependence of near-surface similarity scaling on the anisotropy of atmospheric turbulence. Q. J. R. Meteorol. Soc. (2018) 144:641-657.

[16] Spalart, P.R. and Allmaras, S.R. A one-equation turbulence model for aerodynamic flows, AIAA Paper. (1992) 92-0439.

[17] Bakhvalov, P.A., Abalakin, I.V., and Kozubskaya, T.K. Edge-based reconstruction schemes for unstructured tetrahedral meshes. Int. J. Numer. Methods Fluids (2016) 81(6):331-356.

[18] Abalakin, I.V., Bakhvalov, P.A., Gorobets, A.V., Duben, A.P., and Kozubskaya, T.K. Parallel research code NOISEtte for large-scale CFD and CAA simulations. Vychisl. Metody Programm. (2012) 13(3):110-125.
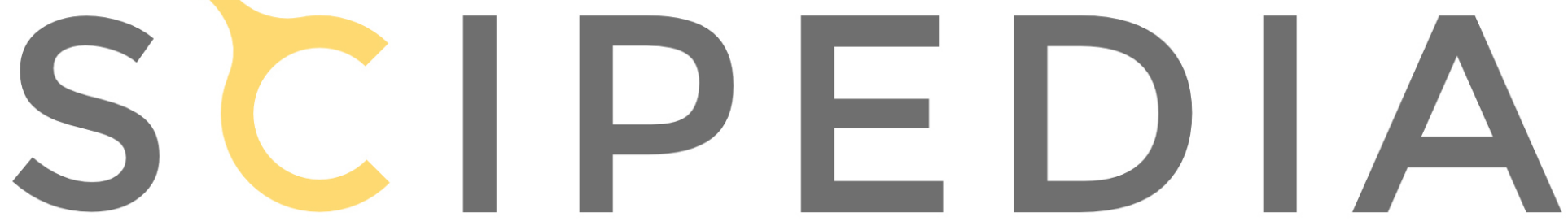

Register for free at https//www.scipedia.com to download the version without the watermark 